\title{
Is there a Histological Profile in the Carcinogenesis of Thyroid Cancer?
}

\author{
Yan Wang and Min Hou* \\ Department of Pathology, Affiliated Cancer Hospital and Institute of Guangzhou Medical University, China
}

Submission: January 17, 2020; Published: February 11, 2020

*Corresponding author: Min Hou, Department of Pathology, Affiliated Cancer Hospital and Institute of Guangzhou Medical University, 78 Hengzhigang Rd, Guangzhou 510095, China

\begin{abstract}
Unlike most of the lining epithelia in which a histological profile from benign proliferation to malignant transformation can be recognized, the pathologic diagnosis of thyroid cancer relies on the characteristic nuclear features in papillary thyroid carcinoma and invasive signs in follicular thyroid carcinoma. There are little, if any, morphological descriptions to guide surgical pathologists to differentiate borderline or precancerous changes from true cancers in their daily practice. In fact, they are facing some difficulty in the diagnosis of certain nodular growth, especially in intraoperative frozen sections. The nuclear atypia is used as a criterion in the cytopathologic evaluation of the Bethesda system for reporting thyroid cytopathology but not in the histological assessment of a thyroid nodule. This manuscript raises a question for discussion. That is, whether a histological profile exists in the carcinogenesis of thyroid cancer. After all, pathologists rely on morphologies to make decisions at the first line.

Keywords: Thyroid cancer; Thyroid nodule; Thyroid goiter; Carcinogenesis; Histology
\end{abstract}

\section{Opinion}

Most of the thyroid cancer originates from follicular cells. Histologically those tumors are mainly classified into papillary thyroid carcinoma (PTC), follicular thyroid carcinoma (FTC) and anaplastic thyroid carcinoma (ATC). PTCs are the most common type and account for $85 \%$ to $90 \%$ of the cases, followed by FTCs with a rate of $5 \%$ to $10 \%$. The broad availability and utility of ultrasound uncovers a large body of thyroid nodules of small size, which eventually leads to the diagnostic approach of fine-needle aspiration (FNA). It is said that the rapid increasing incidence of thyroid cancer is not an endemic of the disease but rather an endemic of the diagnosis as its mortality is low and stable [1]. The practice of thyroid FNA actually brought about the establishment of the Bethesda system for reporting thyroid cytopathology (TBSRTC) in 2007 and was updated to the 2017 revision [2,3]. The system aims to communicate the results of FNA to clinicians by using standardized interpretation of cytopathologic findings. The application of TBSRTC has thrived the molecular testing of thyroid cancer [4].

In turn, molecular analyses reveal that the carcinogenesis of PTC and FPT might go through different pathways [5]. Since follicular variants of PTC are quite indolent and rarely harbor the mutation of BRAFV600E, this type of tumors is reclassified as noninvasive follicular thyroid neoplasm with papillary-like nuclear features (NIFTP) in 2016 [6]. In the perspective of molecular carcinogenesis, it seems that thyroid follicular cells do not need many somatic alterations to become malignant [5,7]. Studies have shown that PTCs are driven by a small group of driver genes and different driver groups lead to different pathologies with distinct signaling and differentiation characteristics and that thyroid follicular adenoma (FTA) is evolved as much as FTC at the genomic level $[7,8]$. Moreover, morphologically benign lesions of thyroid gland may bear key genetic changes in thyroid cancer, such as RET translocations or RAS mutations, with high prevalence $[9,10]$.

Contrary to the advances in molecular biology, the histological criteria used for the diagnosis of a thyroid cancer stay at the place of looking for characteristic nuclear features or invasive signs. Surgical pathologists are still facing a small percentage of thyroid nodules with certain difficulty. Unlike most of the lining epithelia, in which a histological profile from simple proliferation, low-/ high-grade dysplasia towards carcinoma in situ with minimum invasion can be recognized, thyroid follicular cells are unique in the aspect. There are little, if any, morphological descriptions to guide surgical pathologists to differentiate borderline or precancerous changes from true cancers in their daily practice. Since there is 
a different genetic landscape in benign lesions of thyroid gland [11], a tumorous growth with potential malignancy may have to be validated by molecular evaluation.

Here is a case as an example. A 41-years-old male came to our hospital because of a lump in front of his neck discovered by himself by accident. The ultrasonography showed multiple mixed nodules in both lobes with calcification in the left. He had a total thyroidectomy. One of nodules in the left lobe (the left nodule) was shown in Figure 1 and one of nodules in the right lobe (the right nodule) was shown in Figure 2. The left nodule was pathologically diagnosed as PTC though nuclear features were not quite characteristic. The right nodule was pathologically diagnosed as adenomatous goiter. Afterwards, both nodules went through genetic tests by next generation sequencing. BRAFV600E and IDH1N184S were detected in the left nodule; and HRASGln61Arg in the right nodule.

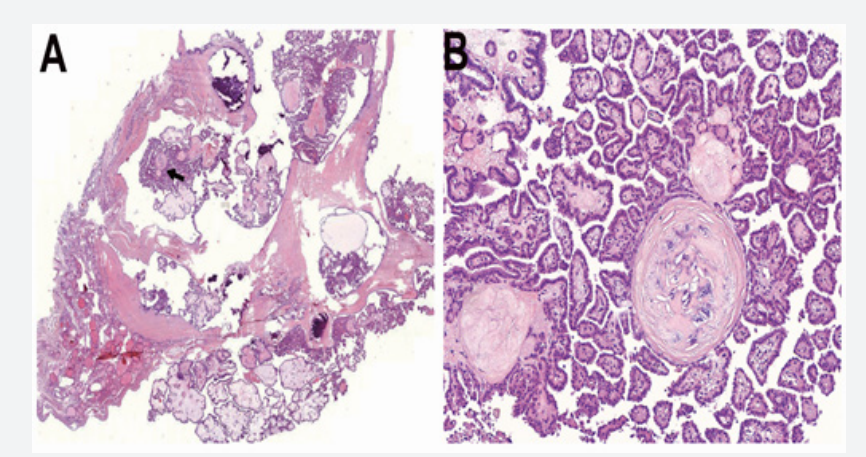

Figure 1: The histology of the nodule in the left lobe of thyroid gland from a 41-years-old man who found his neck lump by accident. (A) The nodule is papillary under lower magnification (a scanned slide, clipped at $0.5 \times$ ). Sclerotic fibrous tissue with calcifications grows into the nodule. The area indicated by an arrow is magnified. (B) Under higher magnification, those papillae emanate directly from large sclerotic cores, mimicking serous borderline tumor of the ovary. Note that the cells covering papillae do not have characteristic nuclei of papillary thyroid carcinoma (clipped at $20 \times$ ).

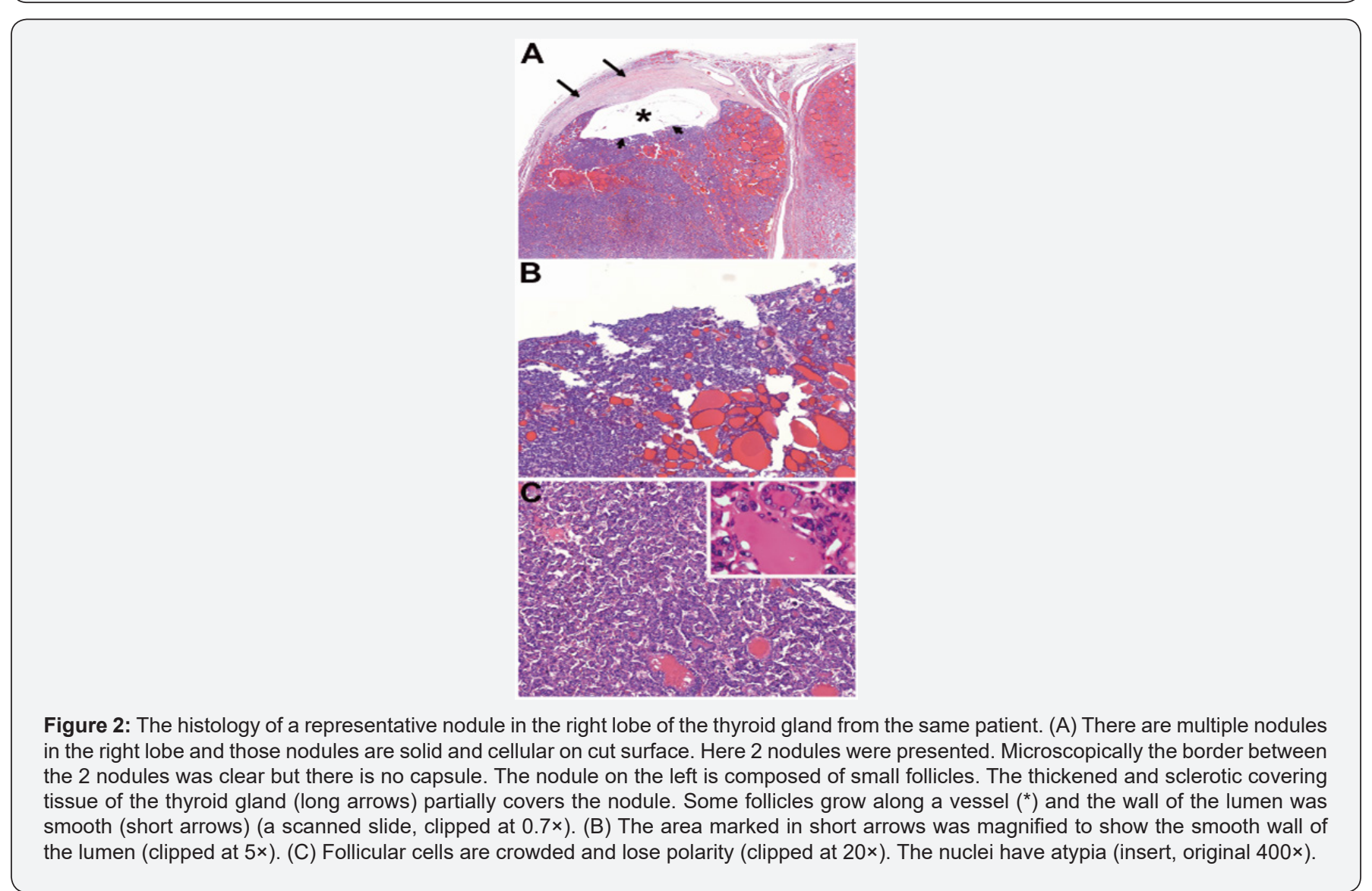


Even with the result of molecular test, the questions that cannot be helped asking are as follows. Is the right nodule a transformed tumorous growth with malignant potential or just a hyperplastic nodule in the basic lesion of thyroid goiter? How about the diagnoses of other nodules with and without similar changes? What should be the proper pathologic diagnosis for the whole pathologic changes of the thyroid gland? How are those nodules in the thyroid gland developed in terms of pathogenesis? Until these questions are answered the authors cannot face the next similar case with confidence.

Histologically, there should be a profile for the tumorous growth of thyroid follicular cells, no matter whether it is in the direction of PTC or FTC. Do morphologies, such as great cellularity, small follicles, degenerative changes, nuclear atypia, loss of nuclear polarity and thickened fibrous tissue on the surface of thyroid glands (thickened capsule), indicate that something more advanced is going on? Is it possible that an FTC arises in nodular thyroid goiter? To date pathologists rely more on immunohistochemical studies and/or molecular tests in order to make up the less confidence in histological diagnosis even though sometimes those techniques are essential. After all, ancillary tests are "aftersensation". During intraoperative diagnosis it is really challenging for pathologists to deal with nodular lesions which require discrimination of a hyperplastic nodule from a tumorous growth with malignant potential.

\section{References}

1. Davies L and HG Welch (2014) Current thyroid cancer trends in the United States. JAMA Otolaryngol Head Neck Surg 140(4): 317-22.
2. Cibas ES, SZ Ali, and NCITFSotS Conference, The Bethesda System For Reporting Thyroid Cytopathology. Am J Clin Pathol 132(5): 658-665.

3. Cibas ES and SZ Ali (2017) The 2017 Bethesda System for Reporting Thyroid Cytopathology. Thyroid 27(11): 1341-1346.

4. Rossi ED, L Pantanowitz, WC Faquin (2019) The Role of Molecular Testing for the Indeterminate Thyroid FNA. Genes (Basel) 10(10).

5. Rita S, Johannes Haybaeck, O Tsybrovskyy (2017) Molecular Aspects of Thyroid Carcinogenesis. In: J Haybaeck (Ed.) Mechanisms of Molecular Carcinogenesis, Springer International Publishing, Switzerland, Europe, Pp. 175-184.

6. Nikiforov YE, Seethala RR, Tallini G, Baloch ZW, Basolo F, et al. (2016) Nomenclature Revision for Encapsulated Follicular Variant of Papillary Thyroid Carcinoma: A Paradigm Shift to Reduce Overtreatment of Indolent Tumors. JAMA Oncol 2(8): 1023-1029.

7. Cancer Genome Atlas Research Network (2014) Integrated genomic characterization of papillary thyroid carcinoma. Cell 159(3): 676-690.

8. Jung SH, Kim MS, Jung CK, Park HC, Kim SY, et al. (2016) Mutational burdens and evolutionary ages of thyroid follicular adenoma are comparable to those of follicular carcinoma. Oncotarget 7(43): 6963869648.

9. Guerra A, Sapio MR, Marotta V, Campanile E, Moretti MI, et al. (2011) Prevalence of RET/PTC rearrangement in benign and malignant thyroid nodules and its clinical application. Endocr J 58(1): 31-38.

10. Angell TE (2017) RAS-positive thyroid nodules. Curr Opin Endocrinol Diabetes Obes 24(5): 372-376.

11. Ye L, Zhou X, Huang F, Wang W, Qi Y, et al. (2017) The genetic landscape of benign thyroid nodules revealed by whole exome and transcriptome sequencing. Nat Commun 8: 15533.

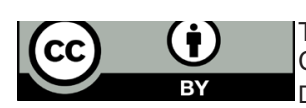

This work is licensed under Creative Commons Attribution 4.0 Licens

DOI: 10.19080/JETR.2020.05.555662 